\title{
Study of Variations in the Origin and Course of Musculocutaneous Nerve
}

\author{
Dr P. D. Sonje ${ }^{* 1}$, Dr P. Gupta ${ }^{2}$, Dr P Vatsalaswamy ${ }^{3}$ \\ ${ }^{* 1}$ Dr D.Y. Patil Medical College Pimpri, Pune, Dept. of Anatomy \\ ${ }^{2,3}$ Dr D.Y.Patil Medical College, Pimpri, Pune
}

\section{$\underline{\text { Abstract- }}$}

Variations in the origin, course,branching pattern, termination and connection of the musculocutaneous nerve are not uncommon.The musculocutaneous nerve arises from the lateral cord of brachial plexus,opposite the lower border of pectoralis minor, pierces the coracobrachialis, descends laterally between the biceps and brachialis to the lateral side of arm and just below the elbow it pierces deep fascia lateral to the tendon of biceps brachii to continue as lateral cutaneous nerve of forearm.The aim was to study the variations in the origin and course of musculocutaneous nerve.Variations like musculocutaneous nerve did not pierce the coracobrachialis muscle, musculocutaneous nerve forming communications with median nerve were found in the present study. Knowledge of these variations is important for surgeons, clinicians and anatomists.Awareness of possible variations is essential to avoid unexpected complications during surgical procedures such as brachial plexus block, arthroscopy of shoulder joint and repair of fractures of humerus.

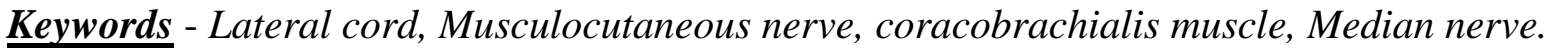

\section{Introduction}

Brachial plexus and their variations are very well known. Musculocutaneous nerve is one of the nerves which showed numerous interesting variations during routine dissection. Hence the present study was conducted and variations in the musculocutaneous nerve were studied. Musculocutaneous nerve arises from the lateral cord (C5-7) of brachial plexus opposite the lateral border of pectoralis minor muscle. It pierces coracobrachialis and descends laterally between biceps and brachialis to the lateral side of the arm. Just below the elbow it pierces the deep fascia lateral to the tendon of biceps, and continues as the lateral cutaneous nerve of the forearm .Musculocutaneous nerve supplies coracobrachialis, both heads of biceps and most of brachialis. The branch to coracobrachialis is given off before the musculocutaneous nerve enters the muscle: its fibres are from the seventh cervical ramus and may branch directly from the lateral cord.Branches to biceps and brachialis leave after the musculocutaneous nerve has pierced coracobrachialis: the branch to brachialis also supplies the elbow joint. The musculocutaneous nerve supplies a small branch to the humerus, which enters the shaft with the nutrient artery. The median nerve has two roots from the lateral (C5,6,7 ) and medial (C8,T1) cords, which embrace the third part of axillary artery, and unite anterior or lateral to it .If the lateral root of median nerve is small, the musculocutaneous nerve (C $5,6,7$ ) connects with median nerve in the arm. (1)

Numerous variations like non piercing musculocutaneous nerve, its communication with median nerve were found in this study. These variations in the musculocutaneous nerve are important in surgical and orthopaedic cases. In previous studies different types of classifications of variations of musculocutaneous nerve are given .In present study the findings were recorded and also compared with previous classification.

Knowledge about these variations is important for surgeons, clinicians and anatomists. Awareness of 
possible variations is essential to avoid unexpected complications during:

- $\quad$ surgical reconstructive procedures such as arthroplasty in recurrent shoulder dislocation,

- in arthroscopy of shoulder joint

- Repair of fractures of humerus.

- performing neurotization of Brachial plexus

- In brachial plexus blocks, so that these structures can be identified and protected. (2)

\section{MATERIALS AND METHODS}

40 cadavers ( 35 male $\& 5$ female) 80 upper limbs were dissected in the Department of Anatomy, Dr D Y Patil Medical College \& Research Centre, Pimpri, Pune to study the variations in the musculocutaneous nerve .The cadavers were embalmed in $10 \%$ formalin. All limbs were meticulously dissected and variations in the musculocutaneous nerve were noted and photographed.

\section{RESULTS}

80 upper limbs (40 rt 40 lt) were dissected to study variations in the musculocutaneous nerve. Following observations were recorded. Total eleven cases showed variation in the origin and course.

Following types of variations were found:

Type 1 - Non piercing coracobrachialis and having two communicating branches with median nerve

Median nerve was formed normally in the axilla by lateral and medial roots.

Branch to the coracobrachialis was given from the lateral cord, instead of musculocutaneous nerve.
Musculocutaneous nerve was given from the lateral cord in the axilla at the level of formation of median nerve.Then it passed down, without piercing the coracobrachialis muscle and in the middle of arm it gave branches to biceps muscle and brachialis muscle. In the arm the course of musculocutaneous nerve was not normal, it was passing along medial side of biceps muscle instead of passing between biceps and brachialis muscle.

There were two communicating branches between musculocutaneous and median nerve.

This finding was seen unilaterally in 3 cases on the left side while in 1 case on right side.

Figure 1 -Non piercing coracobrachialis and having two communicating branches with median nerve.

- LC-lateral cord, MN-median nerve, MCN - musculocutaneous nerve,

- BR TO CB.- branch to coracobrachialis, BR TO BI.- branch to biceps,

- BR TO BR- branch to brachialis, CB communicating branch,

- $\mathrm{CBM}$ - coracobrachialis muscle, BIMbiceps muscle AA- axillary artery.

Type 2 - Non piercing coracobrachialis without communication with median nerve

The musculocutaneous nerve arose from the lateral cord.Branch to the coracobrachialis muscle was given from lateral cord.The musculocutaneous nerve passed along the medial side of the arm without piercing the coracobrachialis muscle .Branches to biceps and brachialis muscle were given in the lower part of the arm and further it passed down to cubital fossa.It also gave lateral cutaneous nerve of forearm. This type of finding was found in 3 cases,two on right side and one on left side . 
Figure 2- Non piercing coracobrachialis without communication with median nerve

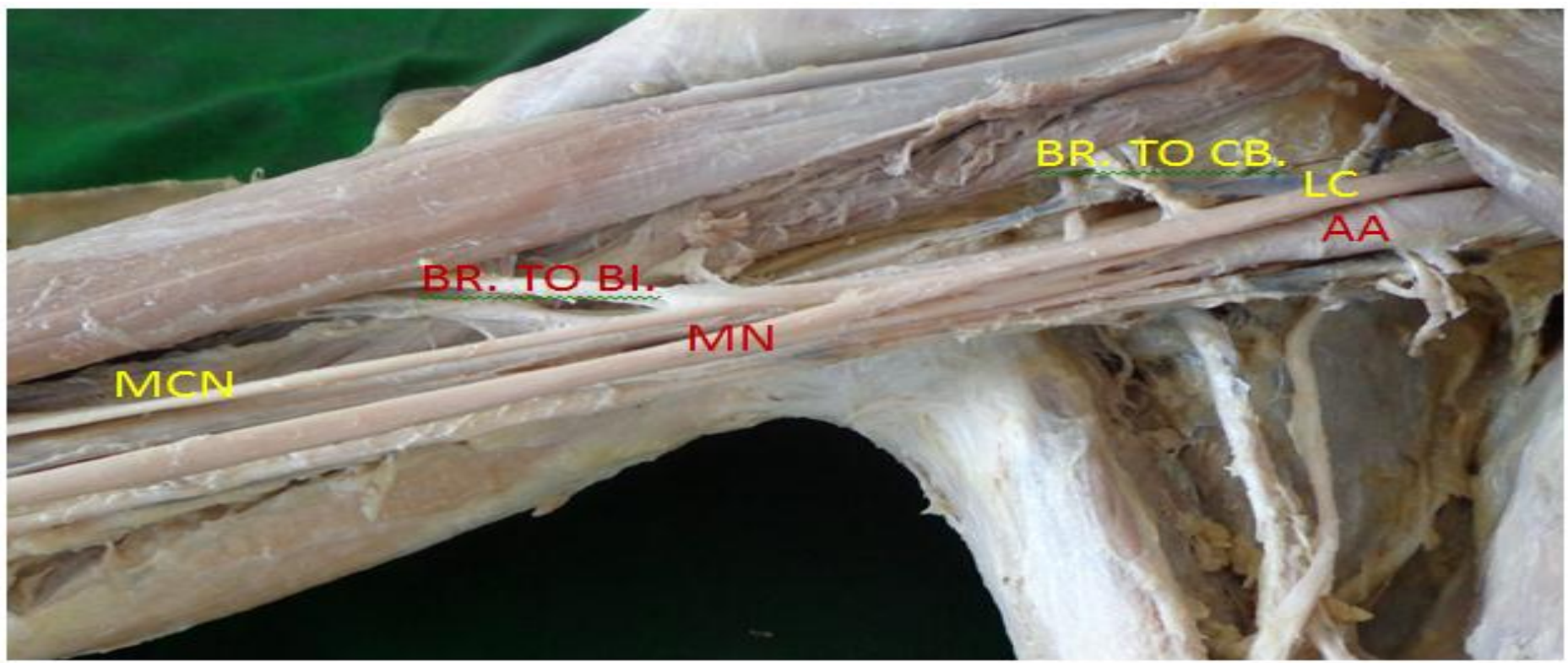

- LC-lateral cord, MN-median nerve, $\mathrm{MCN}$ - musculocutaneous nerve,

- BR TO CB. - Branch to coracobrachialis, BR TO BI. Branch to biceps,

- AA- axillary artery.

Type 3 - Piercing coracobrachialis muscle having two communicating branches with median nerve.

Formation of median nerve and musculocutaneous nerve is normal.
Course of musculocutaneous nerve is also normal.

There was a thick communicating branch between median and musculocutaneous nerve, which was given out from lateral cord at the origin of musculocutaneous nerve and brachial artery was passing between this communicating branch and median nerve. There was one thinner communicating branch between median and musculocutaneous nerve in the middle of arm. This finding was seen unilaterally on the right side in 2 cases.

Figure 3 - Piercing coracobrachialis muscle having two communicating branches with median nerve.

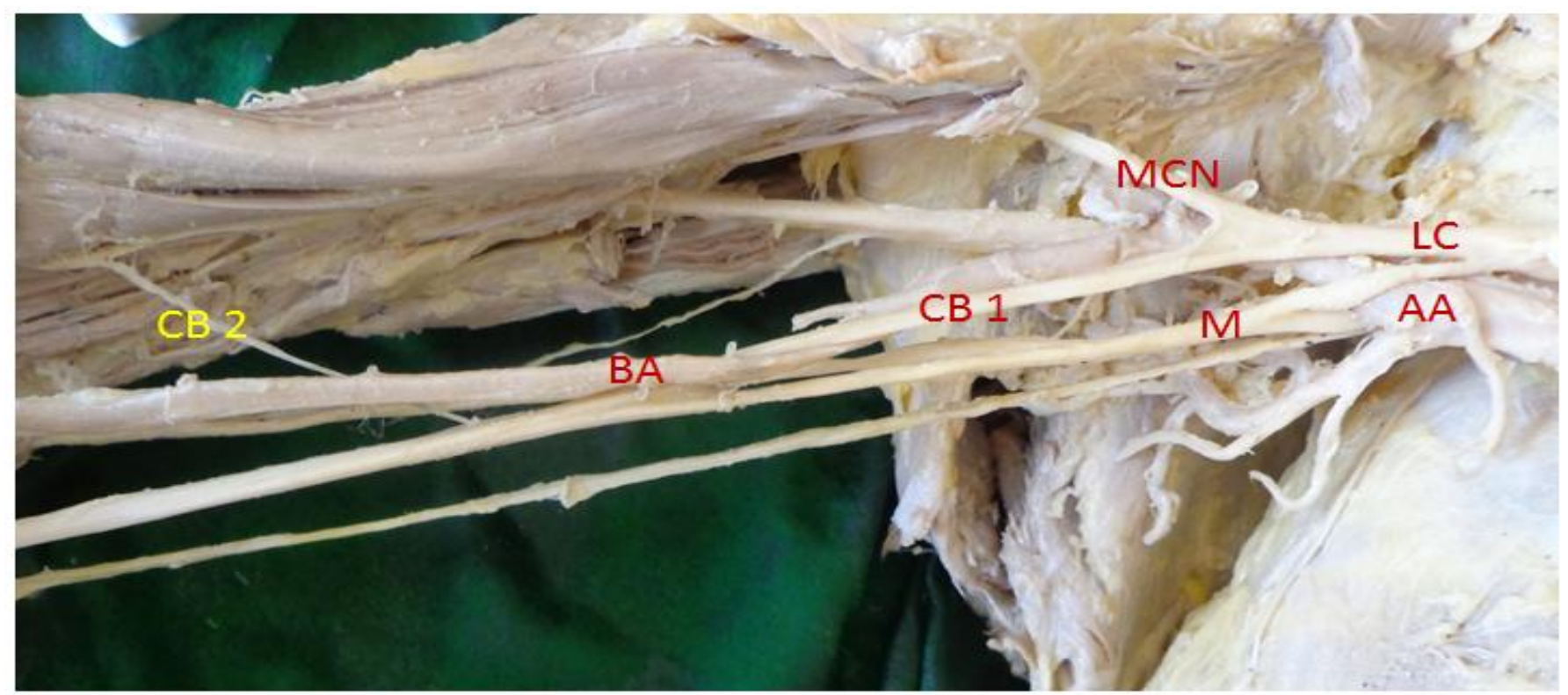


- LC-lateral cord, MN-median nerve, $\mathrm{MCN}$ - musculocutaneous nerve,

- $\mathrm{CB}$-communicating branch, BA- brachial artery, AA-axillary artery.

Type 4- Non piercing coracobrachialis and having one communicating branch with median nerve

Median nerve was formed normally in the axilla by lateral and medial roots but lateral root of median nerve was very thin.

Branch to the coracobrachialis was given from the lateral cord; instead of musculocutaneous nerve. A very thick musculocutaneous nerve was given from the lateral cord in the axilla. Then it passed down, without piercing the coracobrachialis muscle and in the middle of arm giving branches to biceps muscle and brachialis muscle. In the arm the musculocutaneous nerve was passing along medial side of biceps muscle instead of passing between biceps and brachialis muscle.

There was one communicating branch between musculocutaneous and median nerve.

This finding was seen unilaterally in 2 cases, in one case it was on the left side while in 1 case it was on right side.

Figure 4 - Non piercing coracobrachialis and having one communicating branch with median nerve

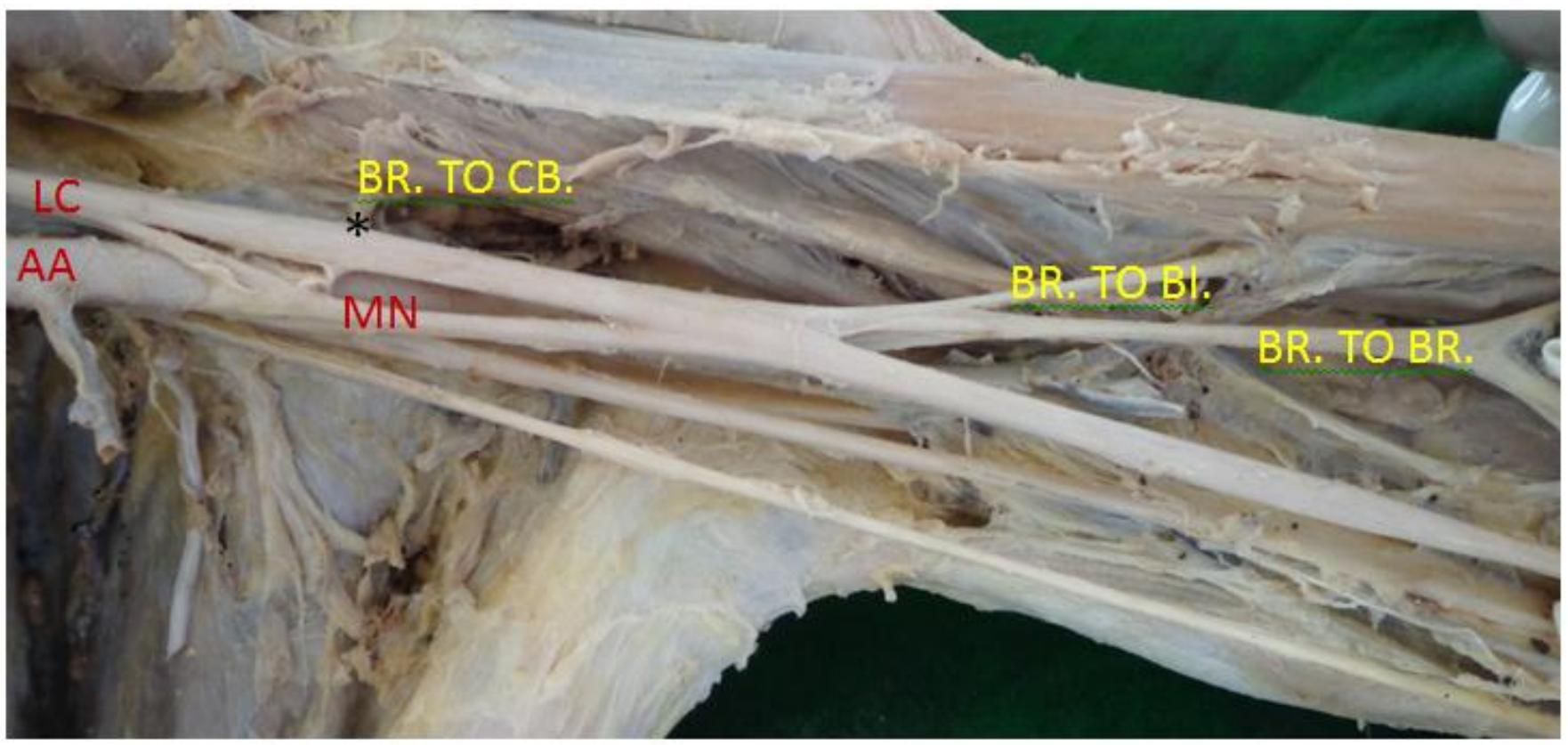

- LC-lateral cord, MN-median nerve, BR TO CB. - Branch to coracobrachialis,

- BR TO BI. - Branch to biceps, BR TO BR- branch to brachialis. 
Diagrammatic representation of findings of the present study is shown in fig (5) Figure 5

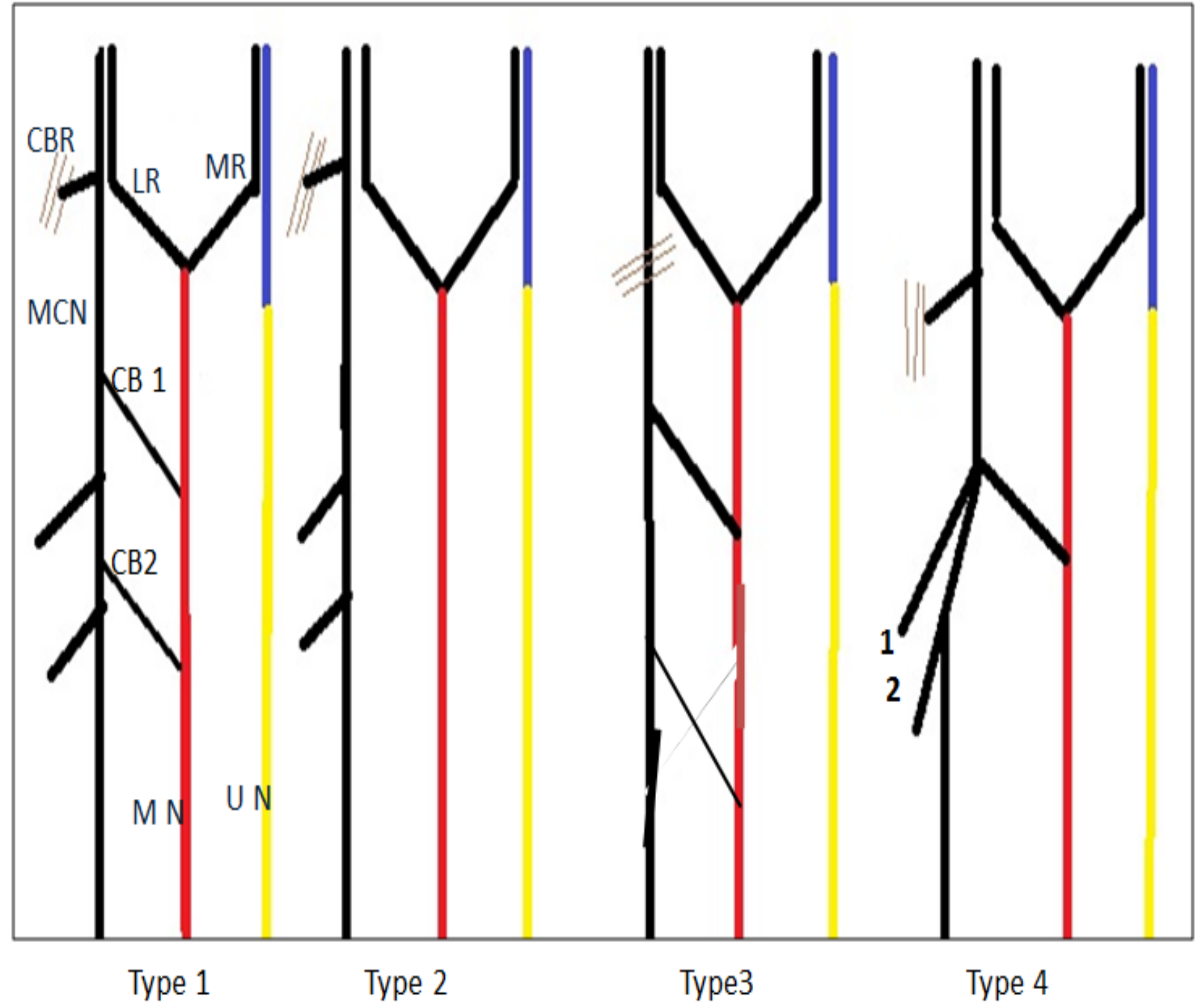

- $\mathrm{CBR}$ - coracobrachialis muscle, LR lateral root, MR - medial root,

- MCN- musculocutaneous nerve, CB1communicating branch 1 ,

- CB2- communicating branch 2 , MNmedian nerve, UN - ulnar nerve,

- 1-branch to biceps, 2- branch to brachialis.

\section{DISCUSSION-}

It is not particularly uncommon to find a nerve trunk of considerable size leaving the musculocutaneous, while this lies behind the biceps and passing distally and medially to join the median nerve. This is to be regarded as a result of median nerve fibers from the lateral cord passing into the musculocutaneous rather than into the lateral root of median, and then rejoining the median nerve at a lower level; when this occurs the lateral root of median nerve is typically small. The communication may be a tiny filament rather than a real nerve trunk. Kerr found that a branch from musculocutaneous nerve to the median had been reported in from $8.1 \%$ to $36.19 \%$ of different series, and estimated its incidence as somewhat less than $24 \%$ found in his series.Hari Rao and Ramchandra Rao reported 28 cases, found among 300 specimens, in which the musculocutaneous nerve did not pierce the coracobrachialis but instead passed between it and the biceps (3).

Raju S et al observed bilateral variations in the anterior compartment of the arm region. In the 
right upper arm the motor branches to the biceps brachii and brachialis muscles found to arise from the branch of the right median nerve, and the same nerve continued as the lateral cutaneous nerve of forearm. This branch was not passing through the coracobrachialis muscle, but this muscle was innervated by direct branch from lateral cord of brachial plexus. They also observed another variation at left side of arm where $\mathrm{MCN}$ and $\mathrm{MN}$ communicated by an abnormal communicating branch which measured about $5 \mathrm{~cm}$ in length. Both the type of variations was found in present study. (4)

Jamuna $\mathrm{M}$ and Amudha $\mathrm{G}$ found absent musculocutaneous nerve in three cases. (5) Bhattarai $\mathrm{C}$ and Poudel PP found numerous types of communications between median and musculocutaneous nerve but in their study the musculocutaneous nerve was piercing the coracobrachialis muscle. It resembles the type 3 of the present study.

Thus this study of unusual course, branching pattern and termination of musculocutaneous nerve is of clinical importance during flap dissections, post traumatic evaluation of the arm or peripheral nerve repair and even for peripheral nerve stimulation in practice of anaesthesia. ${ }^{(6)}$

Sachdeva K. and Singla RK found that musculocutaneous nerve was not piercing the coracobrachialis muscle and it was also forming the communicating channel with median nerve and then branches to the biceps brachii and brachialis muscle were given. This resembles the type 1 classification of the present study.

Huban R Thomas et al found communication between the musculocutaneous nerve and median nerve which resemble type 4 of the present study. (7)

Dr Girish V. Patil and Dr Shishirkumar found musculocutaneous nerve passed along the medial aspect of muscles of arm without piercing coracobrachialis muscle also there was communication between median and musculocutaneous nerve. This resembles type 1 classification of the present study. ${ }^{(8)}$

Remya K., Ashwin Krishnamurthy, Kavitha K. encountered ananomalous communication between musculocutaneous and median nerve in both the upper limbs of a 72 year old male cadaver. The communicating branch arose from the musculocutaneous nerve at a distance of 12.6 $\mathrm{cm}$ and $12.5 \mathrm{~cm}$ from the tip of coracoid process on right and left side and joined the median nerve $16.2 \mathrm{~cm}$ from the same bony point 13 .

Anatomical variations of peripheral nerves have clinical and surgical importance especially in posttraumatic evaluation and exploratory interventions of the arm for peripheral nerve repair and to some extent during flap dissections. Finally, although anterior approach for internal fixation of humeral fractures seems to be safer than the posterior approach because of high risk of radial nerve damage in posterior approach. Thus precise knowledge of variations in musculocutaneous nerve and median nerve may prove valuable in traumatology of the arm, as well as in plastic and reconstructive repair operations. If the surgeons find it necessary to isolate and trace the median nerve and musculocutaneous nerve distally, it is essential to be alert to the communications that may occur between them. ${ }^{(9)}$

MCN has frequent variations. It may run behind the coracobrachialis or adhere for some distance to the median nerve and pass behind the biceps.

Some fibers of the median nerve may run in the musculocutaneous nerve, leaving it to join the proper trunk; less frequently the reverse occurs, the median nerve sending a branch to the musculocutaneous. Occasionally it supplies the pronator teres. ${ }^{(1)}$ 
Incidence of communications between MCN \& MN is shown in the table.

Table 1: Incidence of communications between MCN \& MN

\begin{tabular}{|c|c|c|}
\hline Workers & Year & Percentage \\
\hline Watanabe et al & 1985 & $01.4 \%$ \\
\hline Kosugi et al & 1986 & 21.8 \\
\hline Yang et al & 1995 & 12.5 \\
\hline Venieratos et al & 1998 & 13.9 \\
\hline Rao et al & 2000 & 33.3 \\
\hline Aktan et al & 2000 & 10.4 \\
\hline Choi et al & 2002 & 26.4 \\
\hline Loukas et al & 2008 & 63.5 \\
\hline Guerri et al & 2009 & 53.9 \\
\hline Maeda et al & 2009 & 41.5 \\
\hline
\end{tabular}

Present study showed variations in musculocuaneous nerve in $13.7 \%$ of cases.

\section{Le Minor's Classification}

Type I - No communication between $\mathrm{MCN} \& \mathrm{MN}$

Type II - Some fibers of lateral root of $\mathrm{MN}$ pass thru $\mathrm{MCN} \&$ join $\mathrm{MN}$ in middle of arm
Type III - All fibers of lateral root of MN pass along $\mathrm{MCN}$, after some distance leave it to form lateral root of $\mathrm{MN}$

Type IV - MCN joins lateral root of MN, after some distance $\mathrm{MCN}$ arises from $\mathrm{MN}$ Type $\mathbf{V}-\mathrm{MCN}$ is absent, entire $\mathrm{MCN}$ fibres passes through lateral root of $\mathrm{MN}$ to MN. Fibres to the muscles supplied by MCN come directly from MN. (10)

(MCN - musculocutaneous nerve, $\mathrm{MN}$ - median nerve, U-ulnar nerve.)

Diagram showing Le Minor's classification is shown in fig. 6 Figure 6

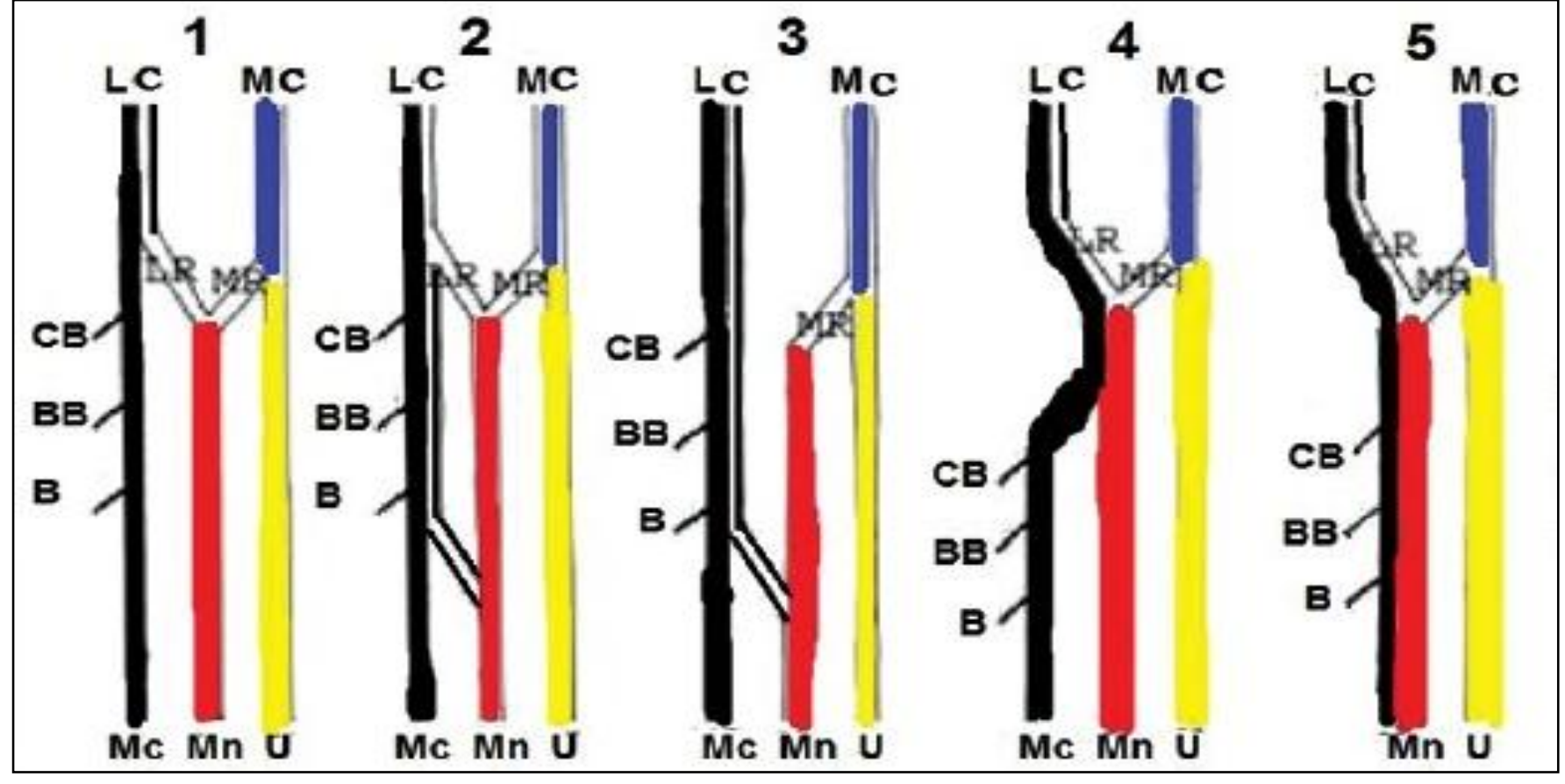


(MCN - musculocutaneous nerve, $\mathrm{MN}$ - median nerve, U-ulnar nerve.)

Venieratos and Anangnostopoulou_suggested classification in relation to coracobrachialis muscle

- Type I: communication is proximal to coracobrachialis muscle

- Type II: communication is distal to muscle;

- Type III: neither the nerve nor the communicating branch pierce the coracobrachialis muscle. (11)

A number of variations in the course and distribution of the musculocutaneous nerve have been reported. Instead of piercing the coracobrachialis muscle, the nerve may adhere to the median nerve for some distance down the arm and then, either as a single trunk or as several branches passes between the biceps and brachialis muscles to supply all three muscles (about $22 \%$ of arms).

Sometimes only a part of the nerve follows this course; this part then rejoins the main trunk after it transmits through and supplies coracobrachialis.

In some cases, instead of the whole trunk of the nerve piercing coracobrachialis, only its muscular branch or only its cutaneous branch pierces the muscle.

The musculocutaneous nerve may be accompanied by fibers from the median nerve as it transmits coracobrachialis; a communicating branch passes from the musculocutaneous to the median nerve. Instead of penetrating coracobrachialis, the nerve may pass behind it or between it and the short head of the biceps muscle. Occasionally, the nerve perforates not only coracobrachialis, but also the brachialis or the short or long head of the biceps muscles.
Very rarely the lateral cord may pierce coracobrachialis and then divide into the musculocutaneous and lateral root of the median nerve (12).

\section{Embryological explanation:}

The limb muscles develop from the mesenchyme of the parietal layer of lateral plate mesoderm during the fifth week of intrauterine life. The axons of the spinal nerves grow distally to reach the muscles and skin. A lack of coordination between the two processes due to altered signalling may lead to the development of multiple variations (17).

\section{CONCLUSION}

Knowledge about variations in these branches of brachial plexus is important for surgeons, clinicians and anatomists. Awareness of possible variations is essential to avoid unexpected complications during surgical reconstructive procedures such as arthroplasty in recurrent shoulder dislocation, in arthroscopy of shoulder joint, repair of fractures of humerus, performing neurotization of Brachial plexus, in brachial plexus blocks, so that these structures can be identified and protected. (14)

\section{ACKNOWLEDGEMENT}

We are very much thankful to our guide, Professor and Dean Dr P Vatsalaswamy for her valuable guidance. We thank to our head of the department Dr P Manvikar for his continous support and guidance for this study. We also thank our Professor Dr V Arole for her guidance. We also thank all non-teaching staff for their co-operation and timely help for this study.

\section{REFERENCES}

[1] HENRY GRAY. The Anatomical Basis of Clinical Practice. 40th edition. Susan Standring, ELSEVIER CHURCHILL LIVINGSTONE ;( 2008): 821. 
[2] Kaur N and Singla R K. Different types of communications between musculocutaneous nerve and median nerve -a cadaveric study in north Indian population. CIBTech Journal of Surgery, 2013, 2 (1); 21-28.

[3] Henry Hollinshead. Anatomy for surgeons. Third edition, vol 3 Harper and Row publishers; (1817): 366.

[4] Raju S,Udaya Kumar P, Ahmed Peer S, Indira Devi B .An Unusual Bilateral Variation of Musculocutaneous Nerve .Journal of Surgical Academia 2013; 3(1):13-15

[5] Jamuna $M$ and Amudha G. A Cadaveric Study on the Anatomic Variations of the Musculocutaneous Nerve in the Infraclavicular Part of the Brachial Plexus. Journal of Clinical and Diagnostic Research. 2011 November (Suppl-1), Vol-5(6): 1144-1147.

[6] Bhattarai C, Poudel PP .Unusual variation in musculocutaneous nerve. Kathmandu University Medical Journal (2009), Vol. 7, No. 4, Issue 28, 408-410.

[7] Huban R THOMAS, Bhagath K POTU, Kumar MR BHAT, Binu MARGARET, Venu MADHA, Stelin WERSELY. Variation in the termination of musculocutaneous nerve Case Report. International Journal of Anatomical Variations (2010) 3: 7879.

[8] Dr Girish V. Patil, Dr Shishirkumar. Musculocutaneous Nerve Not Piercing the Coracobrachialis Muscle and also having Communication with Median Nerve - A Case Report. International Journal of Science and Research Volume 3 Issue 7, July 2014:26-27.

[9] Remya K, Ashwin Krishnamurthy, Kavitha K. Communication between the musculocutaneous and median nerve: occurrence on both the sides A case report. Nitte University Journal of Health Science, December 2011, Vol. I, No.4:55-56.

[10] Le Minor JM. A rare variation of the median and musculocutaneous nerve in man. Arch Anat Histol Embryol.1990; 73:33-42.

[11] Venieratos D, Anagnostopoulou S, Classification of communications between the musculocutaneous and medain nerves. Clin Anat.1998; 11:327-331.

[12] Ronald A. Bergman, Adel K. Afifi, MS, Ryosuke Miyauchi, Illustrated Encyclopedia of Human Anatomic Variation: Opus III: Nervous System: Plexuses Musculocutaneous Nerve.

[13] Chauhan, R. and Roy, T.S Communication Between The Median and Musculocutaneous Nerve- A Case Report. J Anat. Soc. India 51(1) 72-75 (2002).

[14] Guerri-Guttenberg, Roberto A, and Ingolotti M. Classifying musculocutaneous nerve variations. Clinical Anatomy, 2009; 22(6):671683.

[15] Sachdeva K.and Singla RK. Communication between median and musculocutaneous nerve. J.Morphol. Sci., 2011, vol. 28, no. 4, p. 246-249.

[16] Song W .C , Jung H.S, Kim H.J, Shin C, Lee B Y, Koh K S . A Variation of the musculocutaneous nerve absent. Yonsei Med. J. 2003; 44: 1110-1113.

[17] Sadler T W. Langman's medical embryology. Wolters Kluwer Health, $2011 ; 151$. 